\title{
Inhibidores de asociación entre competidores: hipótesis teóricas
}

\author{
ALEJANDRO CASTAÑO RAMÍREZ \\ acastano@poligran.edu.co \\ DOCENTE INVESTIGADOR, GRUPO DE INVESTIGACIÓN EN MERCADEO ESTRATÉGICO \\ POLITÉCNICO GRANCOLOMBIANO
}

\section{RESUMEN}

El desempeño de las personas y de las organizaciones en alianza con un competidor está ligado a los patrones de comportamiento implícitos en la cultura. La disposición de establecer o no, como estrategia de mercados, este tipo de alianzas está condicionada por la cultura. Tres factores de la cultura son centrales para explicar las inhibiciones de las empresas para asociarse con competidores: la confianza, la autoridad y la equidad. La cultura y la empresa en Colombia tienen antecedentes que regulan la manera como funcionan estos factores, antecedentes que suponen inhibiciones a la asociación.

\section{PALABRAS CLAVE}

asociatividad, mercadeo estratégico y cultura empresarial colombiana.

\begin{abstract}
The outcome of employees and organizations in a business alliance is linked to the behavior patterns in a culture. The intention to use a business alliance with a competitor as a marketing strategy is shaped by culture. Three cultural factors are the key to explain cultural inhibitions to this kind of alliances: trust, authority and equity. The culture and the organizations in Colombia have a background driving the way this three factors work towards competitor's business alliances inhibitions.
\end{abstract}

\section{KEY WORDS}

business alliance, strategic marketing, colombian corporate culture.

\begin{abstract}
Alejandro Castaño Ramírez.
Es un antropólogo colombiano dedicado al estudio, análisis e investigación de mercados. Comenzó trabajando etnografía cultural y la descripción de hábitos en agencias de investigación como Napoleón Franco, Quali, Young Marketing y Gloria Gallego Investigación Efectiva, para clientes nacionales e internacionales como Caracol Televisión, Papeles Nacionales, Chevron Texaco, Bavaria y Casa Luker. Posteriormente, Alejandro pasó a trabajar como investigadorconsultor para empresas como GM Colmotores y Meals de Colombia. La realidad empresarial y la consultoría han motivado sus investigaciones académicas sobre el campo del mercadeo, la dinámica interna y externa de las empresas y su relación con las actividades comerciales. Desde el 2007 es docente de investigación de mercados y hace parte del grupo de investigación en Mercadeo Estratégico del Politécnico Grancolombiano.
\end{abstract}

\section{INTRODUCCIÓN}

El estudio de las relaciones asociativas en el campo empresarial es bastante amplio, e incluye muchos tipos de relaciones externas de las empresas: licencias, copropiedad, distribución cruzada, comanufactura, grupos de compra, franquicias, joint ventures, entre otras. En estos diferentes nombres encontramos un concepto central, como lo expresan Meerwarth, Briody y Kulkarni: "las asociaciones son acuerdos colaborativos en los que los aliados combinan recursos, tiempo y experiencia hacia la creación de nuevos conocimientos, productos y servicios". Discovering the Rules: folk knowledge for improving GM partnerships". Greenville: Revista Human Organization, vol 64, num 3, 2005, pág. 287. 
Pero esta gran variedad de acuerdos comerciales no puede ser estudiada en su totalidad en una sola investigación, por lo tanto, es indispensable concentrar la labor de investigación alrededor de uno de estos tipos de acuerdos.

La selección del tipo de acuerdo parte de los propios intereses del grupo de investigación, los cuales se centran en el mercadeo estratégico: por lo tanto, el presente artículo contempla la asociatividad como estrategia de mercadeo, que se materializa en acuerdos comerciales tanto formales como informales entre empresas competidoras en un mercado determinado, "empresas que aceptan participar en los acuerdos en condiciones de participación específicas". Sheth, J. y Parvatiyar, A. "Towards a theory of business alliance formation". Atlanta: Revista Business review, 1 (3), 1992, pág. 75.

Además, es notorio que las publicaciones académicas y no académicas sobre negocios han venido recalcando la importancia de la asociación entre empresas como estrategia efectiva para la sostenibilidad y la supervivencia de los mismos. Pero, aún así, parece que en Colombia esta estrategia no tiene una amplia acogida y en muchos casos tiene poca operatividad. ¿Por qué? ¿Hay alguna inhibición cultural en nuestro país que nos prevenga de asociarnos libremente con otras empresas y de hacer esa asociación exitosa?

El presente presenta una construcción de conocimiento alrededor de la estrategia de mercados, presentando hipótesis acerca de los factores culturales que inhiben la práctica asociativa entre competidores.

La primera parte de éste artículo trata el tema asociatividad y cultura, analizando un ejemplo de interacción cultural. En la segunda parte se expone el modelo de análisis utilizado en la búsqueda de los inhibidores de la asociación. En la tercera parte se hace un análisis de antecedentes y realidades de la cultura y de la empresa en Colombia. Luego se presentan, a manera de conclusión, las hipótesis sobre las inhibiciones de la asociación.

\section{Asociatividad y cultura}

La manera como se establecen las "organizaciones" de personas, entra las que se incluyen obviamente, las alianzas asociativas, obedecen a patrones culturales que son familiares a las personas de un determinado contexto social, de acuerdo con Laura López y Gregorio
Calderón. "Análisis de las dinámicas culturales al interior de un cluster empresarial”. Cali: revista Estudios Gerenciales, Universidad ICESI, No. 99, abril-junio de 2006, pág 20; la “organización” es una construcción subjetiva y los empleados o miembros de una organización le dan significado a lo que hacen, a su trabajo, y al medio ambiente donde se desenvuelven de acuerdo con su acervo cultural, como lo proponen Fons Trompenaars y Charlie Hampdem-Turner. "Riding the waves of culture." New York: Mc Graw Hill Publishers, 1998, pág 7. En la cultura encontramos esos referentes de significado que conforman una organización. Pero la cultura es un concepto antiguo, que ha sido definido de muchas maneras y ha sido aplicado en diferentes contextos, especialmente por la Antropología, contextos que van desde el indígena hasta el empresarial, como identifica Clifford Geertz. "La interpretación de las culturas”. Barcelona: Editorial Gedisa, 1992, pág. 19.

Actualmente la cultura se define como un sistema compartido de significados, que dictamina aquello a lo que se pone atención, la manera en que actuamos y lo que valoramos como propone Trompenaars y HampdemTurner. (Op. Cit, pág. 13).

La importancia de la cultura en fenómenos económicos no ha sido solamente señalada por la Antropología, sino que los mismos economistas han percibido esta importancia; por ejemplo, Michael Porter indica que la cultura de los países tiene influencia en el desempeño económico de los mismos. "La ventaja competitiva de las naciones." Buenos Aires: Javier Vergara Editor, 1991, pág. 109. Esto lo hace evidente estudios realizados sobre asociaciones entre empresas de diferentes países. Uno de estos muestra cómo la interacción comercial entre empleados de México y Estados Unidos está altamente influida por la tendencia hacia el individualismo y el colectivismo. El resultado de la asociación se ve alterado por estas tendencias, en la medida en que, por ejemplo, la responsabilidad se maneja de manera distinta en una cultura individualista, como la estadounidense, donde se tienden a asumir las responsabilidades de manera directa y a hacer implicaciones directas, aunque esto confronte a los miembros de un equipo de trabajo; mientras que, en una cultura colectivista como la mexicana, la responsabilidad se le atribuye menos al individuo y más al grupo de trabajo, con lo cual se evita la confrontación. Los mexicanos tienden a cultivar las 
relaciones a largo plazo entre las empresas por medio de prácticas como la "minimización de errores," mientras que para la cultura empresarial estadounidense las relaciones a largo plazo son menos importantes; se le da mayor importancia a la obtención del beneficio económico, al "confrontar y asumir los errores". Teegen, $\mathrm{H}$ y Doh, J. "U.S/Mexican Business Alliance Negotiations: Impact of culture on authority, trust and performance". Washington D.C: Working paper Series, The center for Latin American Issues, 2002, pág. 21.

Las situaciones resultado de la interacción de dos empresas con características culturales diferentes muestran una tensión dentro de la asociación y pueden marcar futuras inhibiciones o motivaciones. Este ejemplo demuestra que los factores culturales deben ser bien ponderados cuando se trata del tema asociativo.

\section{Modelo de análisis}

Debido a la amplitud del término "cultura" y las amplias y diversas aplicaciones que tiene, se considera necesario delimitar un marco de referencia conceptual. Este ejercicio es de importante reflexión para la Antropología, en la medida en que la disciplina se precia de ser holística. Entonces: ¿por qué cerrar el espacio de compresión y análisis? Y hay una razón: la presente investigación espera ser concluyente. Comenzar un proceso de investigación de manera exploratoria sobre un tema que ha sido ampliamente debatido en la administración, la economía, la psicología organizacional y el mercadeo, sería dejar de lado la vasta construcción de conocimiento alrededor del tema. Por lo tanto, se ha decidido aprovechar este conocimiento y plantear hipótesis a partir del mismo.

En esta búsqueda, encontramos una amplia bibliografía internacional sobre el tema, fruto del trabajo de instituciones sólidas que han ayudado y motivado la asociación comercial, además de producir conocimiento sobre el tema. Se habla de la cultura y se discriminan factores culturales influyentes en el fenómeno.

El primero de estos factores culturales es la confianza; sin duda, el tema más frecuentemente discutido. Las perspectivas sobre la confianza han cambiado con el tiempo, en la medida en que nuevas disciplinas construyen conocimiento sobre nuevos temas.

La manera como las ciencias económicas y administrativas trabajaron el problema de la confianza estaba orientada por una percepción de la confianza como un constructo cuantificable, pero no como una variable que se pudiera deducir de la realidad por sí misma. Para identificar el tipo y el grado de confianza entre dos empresas asociadas comercialmente, se hacía desde una perspectiva que podríamos denominar "reflexiva"; es decir, a través de la evaluación de otras variables que se interpretaban como el resultado de la confianza: la frecuencia de interacción entre empresas, la duración de las relaciones, la reputación. La intensidad de estas variables y su frecuencia dictaminaban el diagnóstico de la confianza en las relaciones comerciales asociativas.

Pero los aportes de la psicología a la economía y a la administración de empresas cada vez ofrece maneras de medir la confianza directamente, a partir de haber desgranado el concepto de confianza. Uno de estos aportes son los hechos por Mike Lombardo y Robert Eichinger quienes discriminan la confianza en: el equipo, en las acciones, en una comunicación confiable. Estos tipos de confianza se miden en una escala de desarrollo, a partir de cada miembro de la organización, clasificando su actitud como una habilidad que puede estar, de acuerdo con una lista de comportamientos, en: no hábil (unskilled), hábil (skilled), sobrecalificado (overused skill). Lombardo, Mike; Eichinger, Robert. "FYI: For Your Improvement, A Guide for Development and Coaching”. Nueva York: Editorial Lominger Ltd Inc, 2004, pág. 14.

La percepción de la confianza se ha complejizado con estas nuevas perspectivas. Una de ellas, encontrada en la búsqueda de bibliografía, fue aquella que explica la confianza en términos no de un individuo, sino en términos de las relaciones que establecen otros. Este nuevo concepto de confianza ha sido investigado a través de herramientas como la Worlwide Values Survey que considera que la confianza tiene diferentes dimensiones: la confianza general en otros y la confianza personalizada.

La primera, la general, se refiere a la confianza que se pone en los individuos de la sociedad, tanto en aquellos a quienes se conoce como en las instituciones. La confianza personalizada, es aquella que solamente tiene lugar entre aquellos que tienen relaciones por periodos de tiempo y especialmente hacia las personas de la familia o los referidos como propone Elvira Salgado. "La Confianza en Colombia". Bogotá: Ediciones Uniandes, pág. 5. 
La bibliografía indica que la confianza en una cultura específica, se establece de acuerdo con la organización social del grupo humano. Las formas de organización social de los grupos humanos han sido caracterizadas en teorías culturales, que utilizan el modelo previamente presentado en este artículo, el modelo colectivistiva/ individualista. Hofstede, Geert. "Cultural constraints in management theories". Filadelfia: revista The Executive, febrero, 1993, vol 7, No 1, pág. 89. La bibliografía reporta que estas tendencias de la cultura tienen un impacto directo en la forma como se da la confianza en un grupo humano, como proponen Kiyonari, Yamagishi, Cook y Cheshire. "Does trust beget trustworthiness? Trust and trustworthiness in Two games and Two Cultures: A research note." Nueva York: revista Social Psychology Quarterly, sep, 2006; 69, 3, pág. 272.

El segundo factor cultural importante que ha sido señalado por los estudios es la autoridad. Esta se entiende como la administración de las actividades de una alianza como proponen Teegen y Doh (Op. Cit. Pág. 9); por ejemplo, en la toma de decisiones, en la formulación de las estrategias empresariales y de mercadeo; en otras palabras, la influencia sobre los participantes de la alianza entre competidores, son las dimensiones de la autoridad.

Las investigaciones sobre la autoridad construyen caracterizaciones de los tipos de alianzas comerciales entre aliados. El ejercicio de la autoridad revela las dinámicas entre las personas y los roles que cada persona asume en la organización. Los roles definen en gran medida los comportamientos de las personas, mucho más cuando están prestablecidos y claros en una organización; además funcionan como símbolos dentro de una organización humana, y permiten a la persona actuar en el interior de esta, estableciendo procedimientos para alcanzar un objetivo, como plantea el sociólogo estadounidense Erwing Goffman. "La presentación de la persona en la vida cotidiana." Buenos Aires: Amorrortu Editorial, 1989, pág. 77.

¿Quién asume los roles con poder de decisión? Es siempre un asunto espinoso, pero nos ayuda a comprender cómo se conforma la asociación; es decir, el tipo de organización de la alianza asociativa entre competidores.

Los estudios sugieren que las alianzas que no tienen un adecuado balance en las relaciones de autoridad tienden a la inestabilidad como proponen Teegen y Doh
(Op. Cit. Pág. 11). Esto refleja que las relaciones de poder, expresadas en el ejercicio de la autoridad, dirigen la vida activa de una alianza.

Al igual que con el factor cultural confianza, como se vive la autoridad por una comunidad humana cambia y es relativa a cada contexto cultural. Se dice que hay culturas con una mayor tendencia al orden, lo que las lleva a comportamientos como la obediencia y al seguimiento de regla como hace evidente el psiquiatra francés Clotaire Rapaille. "El Código Cultural: una manera ingeniosa para entender por qué la gente alrededor del mundo vive y compra como lo hace”. Bogotá: Grupo editorial Norma, 2007, pág. 25. Esta relatividad se ve aumentada por el cambio en las formas de ejercer la autoridad, que cada vez más tienden hacia una "humanización" y salvaguarda de la dignidad humana.

La confianza y la autoridad están relacionadas entre sí, en la medida en que se ha observado que en las alianzas, a mayor confianza, menor necesidad de ejercer la autoridad, como plantean Ranjay Gulati, Nitin Nohria y Akbar Zaheer. "Strategic networks". Chichester, revista Strategic Management Journal, No. 21, 2000, pág. 209.

El tercer factor cultural, que completa el modelo de análisis, es la equidad. Aunque el tema no ha sido frecuentemente discutido en la bibliografía, algunos autores lo consideran central en la constitución de alianzas asociativas con competidores como estrategia de mercadeo. La equidad "es un antecedente de la confianza." Perry, C; Cavaye, A; Coote, L. "Technical and social bonds within business-to-business relationships". Boston: revista Journal of Business and Industrial Marketing. 2002; 17, 1, pág. 78; y permite identificar aspectos con relación a la formación o no formación de una alianza comercial con un competidor, lo cual es un objetivo central en la presente investigación.

La equidad en una asociación es muy importante para su funcionamiento, puesto que la caracterización del comportamiento del asociado, va a dictaminar hasta dónde una determinada organización estará dispuesta a compartir información, recursos y conocimiento. La percepción de un comportamiento oportunista en el aliado llevará a una evaluación de falta de equidad, lo que cambiará la forma de la alianza (Ibid). 


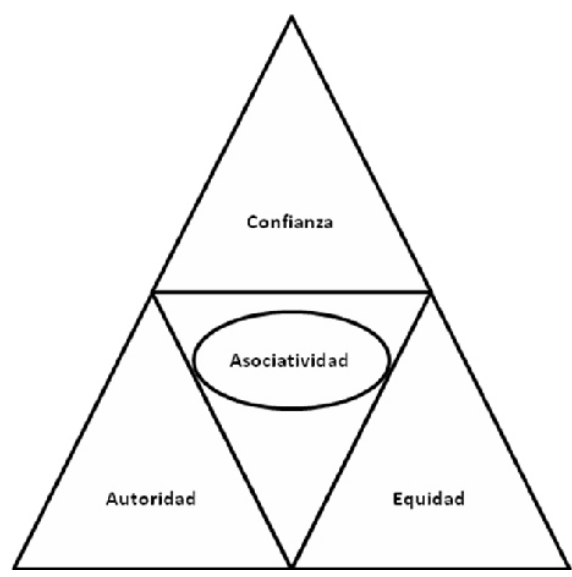

Gráfica no. 1

Modelo cultural de análisis de asociatividad

En la gráfica no. 1 se grafica un modelo de análisis que permitirá comprender y comenzar un camino de construcción de conocimiento alrededor de la relación de la cultura, la asociatividad con competidores y las estrategias de mercadeo. Una investigación con otro enfoque bien podría plantear otro modelo distinto, pero aquí se ha centrado el esfuerzo de análisis sobre estos tres factores porque dentro de cada uno de ellos encontramos hipótesis sobre inhibiciones culturales a la asociatividad entre competidores.

\section{Cultura y empresa en Colombia}

Para poder generar conocimiento sobre los factores culturales de la confianza, la autoridad y la equidad, es necesario identificar algunos patrones de la cultura en Colombia, puesto que ésta es ampliamente diversa y la compresión de su conformación puede explicarnos parte de lo que sucede hoy en día y enriquecer nuestra perspectiva.

Uno de estos elementos es la intención de negar la diversidad presente en Colombia. Un ejercicio de la autoridad. Ejemplo de esto es que solamente desde la Constitución de 1991 las instituciones de la democracia han reconocido que Colombia es un país multicultural y pluriétnico; mientras que ya desde sus comienzos el territorio ha estado poblado por una amplia diversidad (el indígena, el español y el afrodescendiente). Este comportamiento de negar la diferencia señala un ejercicio de la autoridad discriminante que establece jerarquías y esas jerarquías permean los accesos a la riqueza y a los productos.

La identidad de estos círculos sociales se afianzó y las relaciones se fortalecieron alrededor de aquellos "iguales". La primera forma como estas identidades se mostraron en la historia del país fue en las regiones. La fuerza regional se desarrolló en virtud del modelo español de la colonia, que concentró poblaciones alrededor de la búsqueda de oportunidades de negocio específicas como el oro, en la conquista; en la Colonia, el azúcar en el Valle del Cauca, la sal y la papa en la zona cundiboyacense y el arroz en las sabanas de la costa Caribe.

Las identidades regionales se vieron aún más fortalecidas por el aislamiento, puesto que el desarrollo regional de la colonia no tuvo la intención clara y contundente de unir comunicativamente las diferentes regiones y los diferentes mercados. Esto ha devenido en un país altamente fragmentado, con escasas redes de comunicación, como argumenta Emilio Yunis. “¿Por qué somos así? ¿Qué pasó en Colombia? Análisis del mestizaje”. Bogotá: Editorial Temis, 2003, pág. 25.

La tradición aislacionista de los españoles se ha mantenido en periodos posteriores a la Colonia, lo que se demuestra en comportamientos como el abandono de los corredores fluviales y férreos, que en algún momento funcionaban eficazmente, ahora son la prueba fehaciente de que a los dirigentes no les ha interesado mantener el país unido, ni siquiera por vías de comunicación.

La tradición fuerte y arraigada de las regiones, anclada en los ancestros indígena, español y afrodescendiente, ha dado paso de una manera muy parcial, a una cultura nacional integrada, donde solamente la música tropical ha sido el elemento común entre estas culturas regionales, como demuestra el antropólogo Peter Wade. "Música, raza y tradición: música tropical en Colombia”. Bogotá: Editorial Vicepresidencia de la República, 2002, pág. 72. En este panorama, altamente complejo y con mucha distancia, tiene lugar la asociación de empresas en Colombia.

Algunos enfoques investigativos han caracterizado la cultura colombiana como colectivista, lo que significa que el individuo pertenece a pequeños grupos que le ofrecen recompensas por el compromiso de concentrar sus actividades alrededor del grupo y comportarse de acuerdo con sus reglas como argumenta Elvira Salgado (Op. Cit. Pág. 98); es improbable que esto suceda a nivel 
empresarial, puesto la identidad no implica homogeneidad dentro de un grupo; sino que la identidad se concentra, en muchas ocasiones, en la diferencia con otros. De hecho, el tejido empresarial se identifica como ampliamente diverso, con múltiples formas de empresas, desde la micro hasta la multinacional, como expresan López y Calderón (Op. Cit. Pág. 22). La cultura colombiana empresarial tiene una alta distancia institucional, concepto que explica la desvinculación de las empresas a una cadena de valor, como demuestran Gulati, Nohria y Zaheer (Op. Cit. Pág. 208). La presencia de este fenómeno en nuestra cultura empresarial muestra el alto nivel de individualismo en las empresas.

Las empresas en Colombia tienen un alto índice de formación familiar, lo que denota una mayor tendencia hacia la confianza personalizada, concentrada en el círculo familiar.

La cultura también ha venido formulando conceptos sobre los demás y sobre la sociedad en general, un concepto que nos previene a unos contra otros, pensando siempre en la posibilidad de que el otro tenga un comportamiento, lo que nos lleva a medir o escatimar con precisión lo que compartimos. Esta desconfianza generalizada acentúa la identidad de cada grupo dentro del tejido de una cultural colectivista y conlleva a un bajo interés de las empresas por buscar alianzas con la competencia como estrategia de mercadeo. De antemano "sabemos" que "papaya dada, papaya partida". La realidad de estos clichés indica que no es correcto confiar ni compartir con los demás.

Los textos académicos sobre el tema explican que el patrón de comportamiento en Colombia, cuando se hacen negocios con otra persona, se "trata de conseguir el máximo de concesiones y ventajas para sí, sin preocuparse por la situación del otro" y dar concesiones, es decir ceder a lo que el otro pide, es "perder". Ogliastri, Enrique. “¿Cómo negocian los colombianos?” Bogotá: Editorial Alfaomega, 2001, pág. 4.

Este comportamiento es fruto de una cultura que se ha adaptado a la opresión, al elitismo, a la violencia y a la pobreza. El ladinismo es otro término acuñado para describir este patrón cultural, relacionándolo con la figura cultural del héroe, el ladino, que logra burlar la dura sociedad y lograr su propio beneficio, como explica Emilio Yunis. (Op. Cit. Pág. 79).

Dentro de esta cultura encontramos asociaciones negativas como el incumplimiento, la impuntualidad, la evasión de la responsabilidad, la vaguedad, la falta de preparación y el exceso de amabilidad. Además se caracteriza como una cultura emocional, donde las relaciones personales son altamente valoradas porque permiten la flexibilidad en los términos de la negociaciones y la preferencia a pesar de los errores, como manifiesta Enrique Ogliastri (Op. Cit. Pág. 47).

\section{Hipótesis de trabajo}

El análisis de la cultura y la empresa en Colombia nos conduce a tres hipótesis sobre las inhibiciones de la asociación con competidores en Colombia.

La confianza es personalizada e inhibe la asociación comercial entre competidores

La confianza solamente se otorga a quienes están cercanos dentro del grupo de referencia inmediato, como la familia o los conocidos de mucho tiempo, como asevera Elvira Salgado (Op. Cit. Pág. 97), y dar confianza a individuos por fuera de ese círculo se considera peligroso, aunque sea necesaria, forzada por la naturaleza de los negocios en el mundo globalizado.

El individualismo conduce a bajos niveles de tolerancia en el ejercicio de la autoridad.

Hay una resistencia de los empresarios a integrarse a un grupo y actuar grupalmente, como argumentan López y Calderón (Op. Cit. Pág. 34). Se identifica una tendencia al egoísmo, que convella al recelo y al aislamiento. Además, un comportamiento sobreprotector y paternalista rodea la cultura empresarial en Colombia. Estructuras paternales de autoridad implican que el estilo de liderazgo asuma un rol de controlador y supervisor, lo que significa superioridad para quien ejerce el rol de autoridad. El que el líder de una organización acepte la situación inversa, la de ser controlado y supervisado, es percibido como inferioridad y debilidad. Por lo tanto, el trabajo en equipo de una asociación entre competidores se hace difícil. Menos aún cuando los competidores son diferentes, tienen organizaciones de muy diferentes tamaños, y no son iguales.

Aprovechar la situación permanentemente lleva a una percepción automática de oportunismo y falta de equidad. 
La equidad en Colombia no es una realidad frecuente. Investigaciones realizadas demuestran que los colombianos tenemos una cultura distributiva de la negociación, como asevera Enrique Ogliastri (Op. Cit. Pág. 5); que no permite la asociación libre puesto que presupone que el aliado tendrá un comportamiento oportunista, una vez se hayan compartido valores importantes para el negocio.

\section{CONCLUSIONES}

Es claro que los tres factores culturales expuestos previamente tienen antecedentes y raíces culturales que explican teóricamente las inhibiciones a asociarse con los competidores.

La relación entre estos tres factores es también manifiesta. Se identifica la equidad como la piedra angular de una relación de alianza entre competidores. Pero la amplia diversidad en la composición de las empresas en el contexto colombiano, las pone en desigualdad de condiciones frente a la negociación; a lo que se suma que la cultura tiene fuerte influencia en la percepción de una falta de equidad en las relaciones y en las negociaciones; esta influencia es constante e histórica y se caracteriza por una forma de negociación distributiva. Igualmente se observan tendencias culturales a concentrar la confianza en la personalización (familiares y amigos de larga data o, en menor medida, referidos). La percepción de falta de equidad cierra los espacios para que las personas confíen libremente en los otros, en aquellos a quienes recientemente se conoce o que no se conocen, orientando la entrega de la confianza al círculo íntimo. La confianza, vivida de esta forma, conlleva a un crecimiento pausado, que falla para dar resultados en un mundo moderno que está exigiendo algo diferente de los empresarios. Mucho más cuando, por el ámbito de la autoridad, encontramos en la cultura empresarial una fuerte tendencia individualista, donde se recela la información y las prácticas comerciales. Vencer la barrera del recelo implica generar fuertes lazos afectivos entre las personas alrededor de los negocios, lo que toma tiempo. Esta tendencia determina que los empresarios se centren en sus propias prácticas, sin considerar el mundo o sus mejores prácticas. En estas estructuras pequeñas, con estructuras jerárquicas, la respuesta a la autoridad es negativa, puesto que el líder de la organización pretende tener el control de su organización. Compartir ese control, compartir la autoridad, significa debilidad. Asimismo se determina que el individualismo impide la buena disposición para el trabajo en equipo, debido a la tendencia proteccionista que proviene de paternalismo, en el que la autoridad es vertical y pocas veces se comparte horizontalmente.

Este entramado complejo cultural de significados afecta teóricamente el desarrollo de una economía más sólida, en la medida en que inhibe la asociación en general y entre competidores, retrasando la formación de redes de producción integradas que puedan responder a la demandas de producción, a la calidad del mercado internacional actual y de un consumidor cada vez más exigente.

La comprobación de estas hipótesis en el terreno es el paso a seguir en la investigación. Con la investigación de estos tres factores podremos comprender mejor la dinámica de inhibición y plantear opciones de trabajo sobre estos factores para la superación de estas inhibiciones. 


\section{BIBLIOGRAFÍA}

-Hofstede, Geert. "Cultural constraints in management theories", en Filadelfia: The Executive. vol 7, núm.1, febrero, 1993.

-Geertz, Clifford. La interpretación de las culturas. Barcelona: Editorial Gedisa, 1992.

- Goffman, Erwing. La presentación de la persona en la vida cotidiana. Buenos Aires: Amorrortu Editorial, 1989.

-Gulati, Ranjay; Nohria, Nitin; Zaheer, Akbar. "Strategic networks”, en Chichester: Strategic Management Journal. Vol. 21, 2000, pág. 203-215.

-Lombardo Mike y Eichinger, Robert. FYI: For Your Improvement, A Guide for Development and Coaching. Nueva York: Editorial Lominger Ltd Inc., 2004.

-López, Laura y Calderón, Gregorio. "Análisis de las dinámicas culturales al interior de un clúster empresarial”, en Cali: Revista Estudios Gerenciales. Universidad ICESI, núm. 99, abril-junio de 2006.

-Meerwarth, Tracy, Briody, Elizabeth y Kulkarni, Devadatta. "Discovering the Rules: folk knowledge for improving GM partnerships", en Greenville: Human Organization, vol. 64, num. 3, 2005.

-Ogliastri, Enrique. ¿Cómo negocian los colombianos? Bogotá: Editorial Alfaomega, 2001.

-Perry, Chad; Cavaye, Angele y Coote, Len. "Technical and social bonds within business-to-business relationships", en Boston: Journal of Business and Industrial Marketing, 2002.

-Porter, Michael. La ventaja competitiva de las naciones. Javier Vergara Editor. Buenos Aíres, 1991.

-Salgado, Elvira. La Confianza en Colombia. Ediciones Uniandes. Bogotá, 2005.

-Sheth, Jagdish y Parvatiyar, Atul. "Towards a theory of business alliance formation. En: Scandinavian International", en Atlanta: Business review., Vol1, núm. 3, 1992, pág. 71-87.

- Rapaille, Clotaire. El Código Cultural: una manera ingeniosa para entender por qué la gente alrededor del mundo vive y compra como lo hace. Bogotá: Grupo editorial Norma, 2007.

-Teegen, Hildy y Doh, Jonathan. "U.S/Mexican Business Alliance Negotiations: Impact of culture on authority, trust and performance", en Washington D.C.: Working paper Series. The center for Latin American Issues, 2002.
-Teegen, Hildy y Doh, Jonathan. "U.S/Mexican Business Alliance Negotiations: Impact of culture on authority, trust and performance", en Washington D.C.: Working paper Series. The center for Latin American Issues, 2002.

-Trompenaars, Fons y Hampdem-Turner, Charlie. Riding the waves of culture. New York: Mc Graw Hill Publishers, 1998.

-Wade, Peter. Música, raza y tradición: música tropical en Colombia. Bogotá: Editorial Vicepresidencia de la República, 2002.

-Kiyonari, Toko; Yamagishi, Toshio; Cook, Karen y Cheshire, Coye. "Does trust beget trustworthiness? Trust and trustworthiness in Two games and Two Cultures: A research note", en Nueva York: Social Psychology Quarterly, septiembre 2006, pág. 3, 69.

-Yunis, Emilio. ¿Por qué somos así? ¿Qué pasó en Colombia? Análisis del mestizaje. Bogotá: Editorial Temis, 2003. 